\title{
Role of Disorder in the Thermodynamics and Atomic Dynamics of Glasses
}

\author{
A. I. Chumakov, ${ }^{1, *}$ G. Monaco, ${ }^{2,1}$ A. Fontana, ${ }^{2,3}$ A. Bosak, ${ }^{1}$ R. P. Hermann, ${ }^{4,5}$ D. Bessas, ${ }^{4,5, \dagger}$ B. Wehinger, ${ }^{1}$ \\ W. A. Crichton, ${ }^{1,}$ M. Krisch, ${ }^{1}$ R. Rüffer, ${ }^{1}$ G. Baldi, ${ }^{6}$ G. Carini Jr., ${ }^{7}$ G. Carini,${ }^{8}$ G. D’Angelo, ${ }^{8}$ E. Gilioli, ${ }^{6}$ \\ G. Tripodo, ${ }^{8}$ M. Zanatta, ${ }^{9,3}$ B. Winkler, ${ }^{10}$ V. Milman, ${ }^{11}$ K. Refson, ${ }^{12}$ M. T. Dove, ${ }^{13}$ N. Dubrovinskaia, ${ }^{14}$$$
\text { L. Dubrovinsky, }{ }^{15} \text { R. Keding, }{ }^{16} \text { and Y.Z. Yue }{ }^{17, \$}
$$ \\ ${ }^{1}$ European Synchrotron Radiation Facility, F-38043 Grenoble, France \\ ${ }^{2}$ Dipartimento di Fisica, Università di Trento, I-38123 Povo, Trento, Italy \\ ${ }^{3}$ IPCF-CNR, UOS di Roma, clo Roma University La Sapienza, I-00185 Roma, Italy \\ ${ }^{4}$ Jülich Centre for Neutron Science JCNS and Peter Grünberg Institut PGI, JARA-FIT, Forschungszentrum Jülich GmbH, \\ D-52425 Jülich, Germany \\ ${ }^{5}$ Faculté des Sciences, Université de Liège, B-4000 Liège, Belgium \\ ${ }^{6}$ IMEM-CNR, Area delle Scienze, I-43124 Parma, Italy \\ ${ }^{7}$ IPCF-CNR, UOS di Messina, Viale F. Stagno d'Alcontres 37, I-98158 Messina, Italy \\ ${ }^{8}$ Dipartimento di Fisica e Scienze della Terra, Università di Messina, Viale F. Stagno d'Alcontres 31, I-98166 Messina, Italy \\ ${ }^{9}$ Dipartimento di Fisica, Università di Perugia, I-60123 Perugia, Italy \\ ${ }^{10}$ Geowissenschaften, Goethe-Universität, Altenhoeferallee 1, D-60438, Frankfurt a.M., Germany \\ ${ }^{11}$ Accelrys, 334 Cambridge Science Park, Cambridge CB4 OWN, United Kingdom \\ ${ }^{12}$ STFC Rutherford Appleton Laboratory, Chilton, Didcot Oxfordshire OX11 OQX, United Kingdom \\ ${ }^{13}$ Materials Research Institute and School of Physics and Astronomy, Queen Mary University of London, \\ Mile End Road, London E1 4NS, United Kingdom \\ ${ }^{14}$ Material Physics and Technology at Extreme Conditions, Laboratory of Crystallography, University of Bayreuth, \\ D-95440 Bayreuth, Germany \\ ${ }^{15}$ Bayerisches Geoinstitut, Universität Bayreuth, D-95440 Bayreuth, Germany \\ ${ }^{16}$ Max Planck Institut for the Science of Light, D-91058 Erlangen, Germany \\ ${ }^{17}$ Section of Chemistry, Aalborg University, DK-9000 Aalborg, Denmark
}

(Received 28 May 2013; published 15 January 2014)

\begin{abstract}
We measured the density of vibrational states (DOS) and the specific heat of various glassy and crystalline polymorphs of $\mathrm{SiO}_{2}$. The typical (ambient) glass shows a well-known excess of specific heat relative to the typical crystal ( $\alpha$-quartz). This, however, holds when comparing a lower-density glass to a higherdensity crystal. For glassy and crystalline polymorphs with matched densities, the DOS of the glass appears as the smoothed counterpart of the DOS of the corresponding crystal; it reveals the same number of the excess states relative to the Debye model, the same number of all states in the low-energy region, and it provides the same specific heat. This shows that glasses have higher specific heat than crystals not due to disorder, but because the typical glass has lower density than the typical crystal.
\end{abstract}

DOI: 10.1103/PhysRevLett.112.025502

PACS numbers: 63.20.-e, 07.85.-m, 76.80.+y

The low-temperature thermodynamic properties of glasses are accepted to be anomalously different from those of crystals due to the inherent disorder of the glass structure. At temperatures of $\sim 10 \mathrm{~K}$, the specific heat of glasses shows an excess relative to that of the corresponding crystals. The excess specific heat is related to a distinct feature in the spectrum of the atomic vibrations: At frequencies of $\sim 1 \mathrm{THz}$, glasses exhibit an excess of states above the Debye level of the acoustic waves, the socalled "boson peak." The excess of specific heat and the boson peak are universally observed for all glasses and by all relevant experimental techniques. However, the results still do not converge to a unified answer to how disorder causes these anomalies.

The majority of the models explain the boson peak by appealing to various glass-specific features. These include low-energy optical modes [1], onset of mechanical instability related to saddle points in the energy landscape [2] or to jamming [3-5], local vibrational modes of clusters [6] or locally favoured structures [7], librations [8] or other coherent motions [9] of molecular fragments, crossover of local and acoustic modes [10], quasilocal vibrations of atoms in an anharmonic potential [11], broadening of vibrational states in the Ioffe-Regel crossover regime [12], spatial variation of the elastic moduli [13], breakdown of the continuum approximation $[14,15]$, and topologically diverse defects [16], to cite the most important ones.

Alternatively, the boson peak is identified as the counterpart of the acoustic van Hove singularities of crystals, i.e., explained by the piling up of the vibrational states of the acousticlike branches near the boundary of the pseudoBrillouin zone [17-20].

Diverging in explanations of the boson peak, all models agree that the excess states and the excess specific heat of 
glasses are anomalies caused by disorder. The results presented below question this issue.

We studied the atomic dynamics of various glassy and crystalline polymorphs of $\mathrm{SiO}_{2}$. This is not only the archetypical system which established the problem of the boson peak. More important, $\mathrm{SiO}_{2}$ exists in various glassy and crystalline polymorphs, which allows for an analysis of the atomic dynamics as a function of local structure and density. Different to many previous works, the densities of states were measured in absolute units. This allows for a quantitative comparison of the numbers of states for various polymorphs.

The density of states was measured by two techniquesnuclear resonant analysis of inelastic x-ray scattering (NRAIXS) [21] and inelastic x-ray scattering with crystal optics (IXS) [22]. The absolute scale of the DOS was verified by comparing the specific heat calculated from the derived DOS to the measured specific heat for each studied polymorph. For crystals, the reliability of the DOS was further confirmed by ab initio lattice dynamics calculations using density functional perturbation theory [23]. The details of the experimental methods and calculations are reported in the Supplemental Material [24].

Figure 1 shows the density of vibrational states for the ambient and the densified polymorphs of silica glass; Fig. 2 shows those for polycrystalline $\alpha$-cristobalite, $\alpha$ quartz, and coesite. The total (i.e., statistical and systematic) relative uncertainties of the presented DOS are 6\% and $13 \%$ for NRAIXS and IXS data, respectively [24]. The agreement of the data obtained by the two IXS techniques [Figs. 1(a)-(d), Figs. 2(a)-(f)] and the consistency of the calculated specific heat with the experimental specific heat [Figs. 1(e)-(f), Figs. 2(g)-(i)] evidence the reliability of the obtained results.

Usually the comparison of thermodynamic and atomic dynamic properties of glasses and crystals is performed for the most common polymorphs, which for $\mathrm{SiO}_{2}$ are ambient silica glass and $\alpha$-quartz. Such a comparison is shown in Figs. 3(a)-(c). It illustrates the puzzle which established the problem of the boson peak: The DOS of the glass is significantly softer than that of the crystal [Fig. 3(a)], the reduced DOS shows the distinct peak at $\sim 5 \mathrm{meV}$ where the reduced DOS of the crystal is nearly flat [Fig. 3(b)], and at $\sim 10 \mathrm{~K}$ the specific heat of the glass shows an excess over that of the crystal [Fig. 3(c)]. This trend is observed for nearly all glasses. According to the generally accepted point of view, it is attributed to disorder.

However, the other $\mathrm{SiO}_{2}$ polymorphs studied here cast doubts on this conclusion. For instance, the comparison of densified silica glass and $\alpha$-cristobalite [Figs. 3(d)-(f)] would lead to the opposite suggestion that disorder shifts the vibrational states to higher energy [Fig. 3(d)], makes the reduced DOS of the glass nearly flat at the position where the reduced DOS of the crystal has a distinct peak [Fig. 3(e)], and provides the deficit of specific heat at $\sim 10 \mathrm{~K}$ [Fig. 3(f)]. Although this
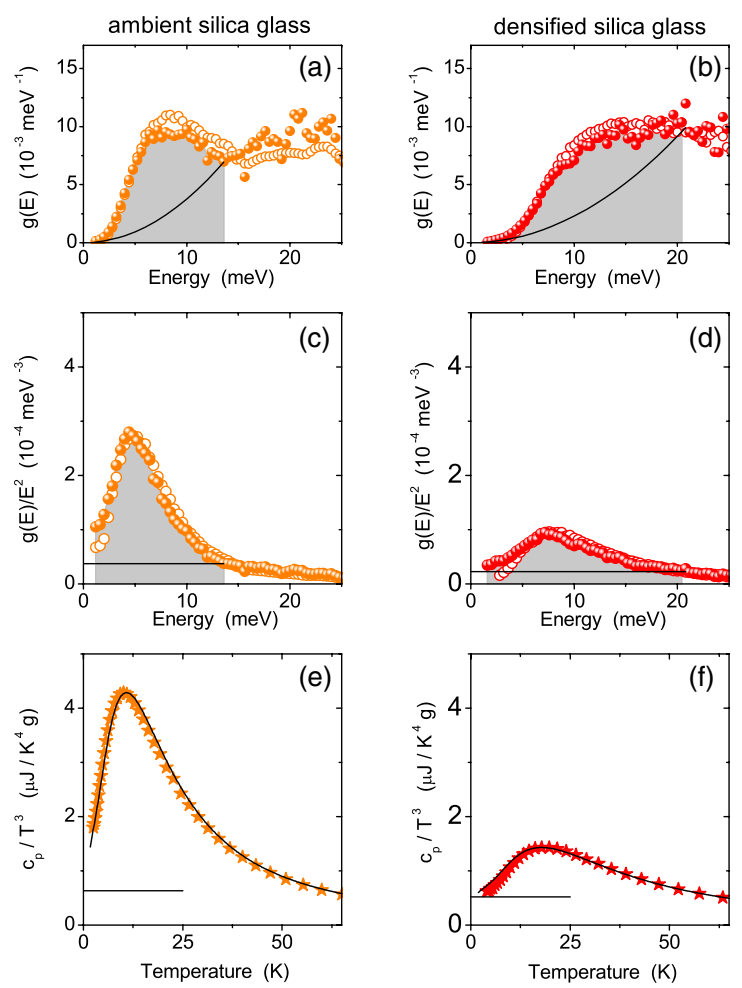

FIG. 1 (color online). The density of states (a)-(b) and the reduced density of states (c)-(d) of ambient and densified silica glass. The solid and the open circles refer to the NRAIXS and IXS data, respectively. The solid lines show the Debye levels for the DOS (a)-(b) and reduced DOS (c)-(d). The area of the filled region of the DOS above the Debye parabola in (a)-(b) gives the number of the excess states. The entire area of the filled region in (a)-(b) gives the number of all states within the same energy ranges. The filled areas in (c)-(d) show the same energy regions for the reduced DOS. (e)-(f) The measured specific heat (symbols) and the specific heat calculated from the corresponding DOS (solid lines). The horizontal lines in (e)-(f) show the Debye levels for the specific heat.

opposite trend is rare, it was observed, e.g., for amorphous and crystalline zeolite $[27,28]$.

A check of the densities of the various $\mathrm{SiO}_{2}$ polymorphs (Table 1) reveals that Figs. 3(a)-(c) compare a low-density glass with a high-density crystal, whereas Figs. 3(d)-(f) do the opposite-compare a high-density glass with a lowdensity crystal (as for amorphous and crystalline zeolite $[27,28])$. A different density is accompanied by different sound velocity [29], which determines different properties of the elastic medium of the glass and influences its atomic dynamics $[20,30,31]$. Therefore, in order to reveal the effect of disorder unambiguously, a proper comparison should be done for glassy and crystalline polymorphs with matched densities, i.e., for ambient silica and $\alpha$-cristobalite (lowdensity polymorphs) and for densified silica and $\alpha$-quartz (high-density polymorphs). Sound velocities, Debye energies, and diffraction patterns [24] show that these pairs indeed have close elastic properties and local structures. 

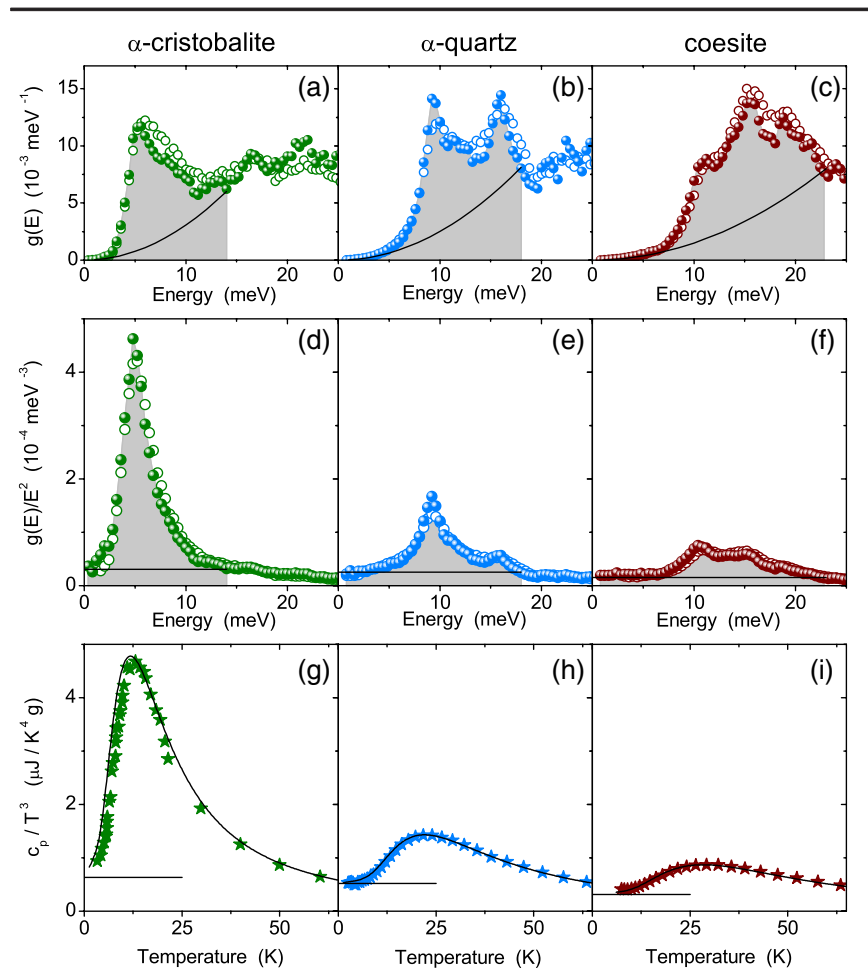

FIG. 2 (color online). The density of states (a)-(c) and the reduced density of states (d)-(f) of $\alpha$-cristobalite, $\alpha$-quartz, and coesite. (g)-(i) The measured specific heat (symbols) of $\alpha$-cristobalite (from Refs. [25,26]), $\alpha$-quartz, and coesite and the specific heat calculated from the corresponding DOS (solid lines). The symbols, solid lines, and the filled regions have the same meaning as in Fig. 1.

A comparison of $\mathrm{SiO}_{2}$ glasses and crystals with matched densities (Fig. 4) reveals a simple but quite unexpected picture. The density of states of the glass does not differ much from that of the crystal except for some smearing out of the DOS features [Figs. 4(a),(d)], the peaks of the reduced DOS are located nearly at the same energies Figs. 4(b),(e)], and the specific heat is practically the same for the glassy and crystalline counterparts [Figs. 4(c),(f)].

In essence, Fig. 4 shows that the excess of specific heat of the typical (ambient) silica glass relative to the typical ( $\alpha$ quartz) crystal [(Fig. 3(c)] is related not to the structural disorder of the glass but to its lower density. Note that the lower density of glasses relative to crystals is not limited to the $\mathrm{SiO}_{2}$ system but is a general trend well documented in the literature (often discussed as the excess volume of glasses).

The similarities of glasses and crystals with matched densities are further confirmed by a quantitative analysis of the number of states. The solid lines in Figs. 1(a)-(d) and Figs. 2(a)-(f) show the Debye levels calculated from density and sound velocities data [24]. The area of the filled region of the DOS above the Debye parabolas in Figs. 1(a)-(b) and Figs. 2(a)-(c) gives the number of the excess states $N_{e x}$. The entire area of the filled region gives the number of all states $N_{\text {all }}$ within the same energy range. ambient glass vs $\alpha$-quartz

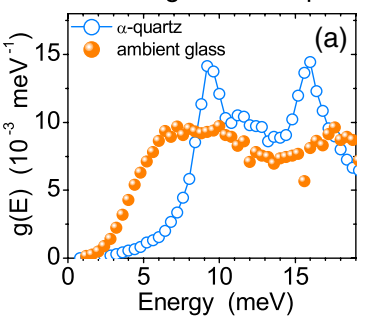

densified glass vs $\alpha$-cristobalite
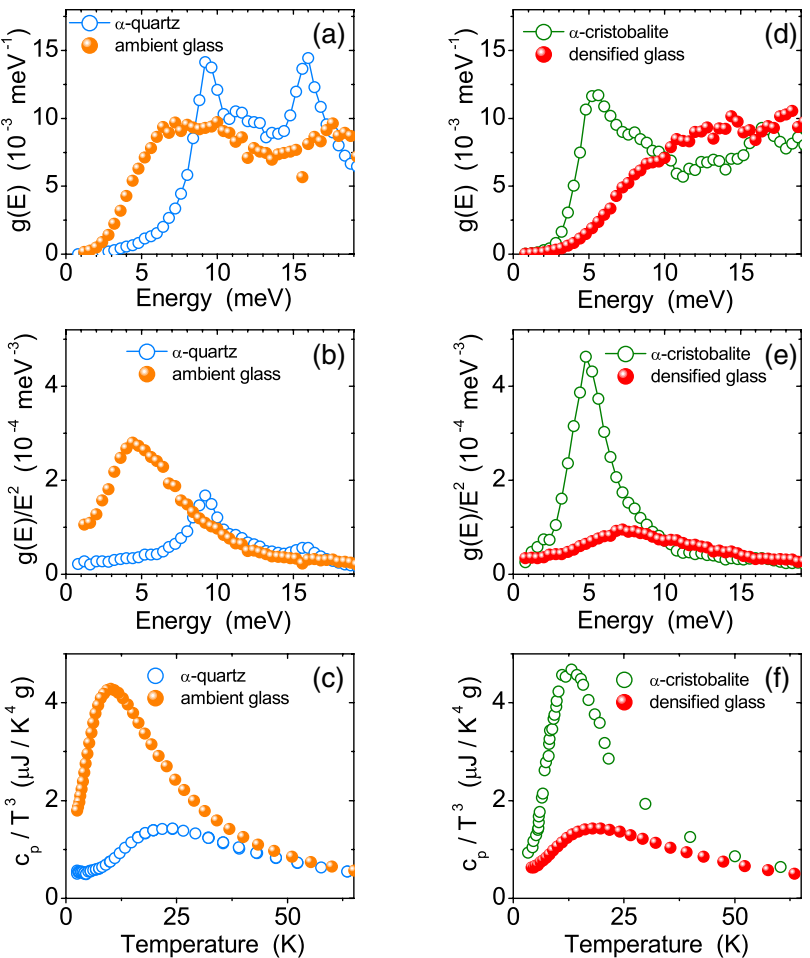

FIG. 3 (color online). Comparison of the density of states (a), (d) and the reduced density of states (b),(e) obtained with the NRAIXS technique, and of the specific heat (c),(f) for ambient silica glass and $\alpha$-quartz (a)-(c), and for densified silica glass and $\alpha$-cristobalite (d)-(f). The experimental data for the specific heat of $\alpha$-cristobalite in (f) are those from Refs. [25,26].

Table 1 shows that the excess of states of silica glasses is not remarkable relative to their crystalline counterparts. It is about $5 \%-6 \%$ for both glassy polymorphs, which is nearly the same as the excess of states for $\alpha$-cristobalite and $\alpha$ quartz crystals $(6 \%-7 \%)$ and even less than that for coesite (9\%). These observations cannot be caused by an accidental underestimation of the excess states for the glasses: The analysis of the inelastic neutron scattering data for ambient

TABLE I. The number of the excess states $N_{e x}$ in the DOS of glassy and crystalline polymorphs of $\mathrm{SiO}_{2}$, the number of all states $N_{\text {all }}$ within the same energy regions derived from the NRAIXS data, and the fraction of the acoustic states $N_{a c}$ for the three crystals given by $N_{a c}=N^{-1}$, where $N$ is the number of atoms in a primitive unit cell of $\alpha$-cristobalite $(N=12), \alpha$ quartz $(N=9)$, and coesite $(N=24)$. The numbers of states are given as a percentage of the unit area of the DOS.

\begin{tabular}{ccccc}
\hline \hline Sample & Density $\left(\mathrm{g} / \mathrm{cm}^{3}\right)$ & $N_{e x}(\%)$ & $N_{\text {all }}(\%)$ & $N_{a c}(\%)$ \\
\hline Ambient silica glass & 2.20 & $5.3(3)$ & $8.4(5)$ & $\ldots$ \\
$\alpha$-cristobalite & 2.29 & $5.6(3)$ & $8.4(5)$ & 8.333 \\
Densified silica glass & 2.67 & $5.9(4)$ & $12.8(8)$ & $\ldots$ \\
$\alpha$-quartz & 2.65 & $6.6(4)$ & $11.5(7)$ & 11.111 \\
Coesite & 2.93 & $8.6(5)$ & $14.6(9)$ & 4.167 \\
\hline \hline
\end{tabular}



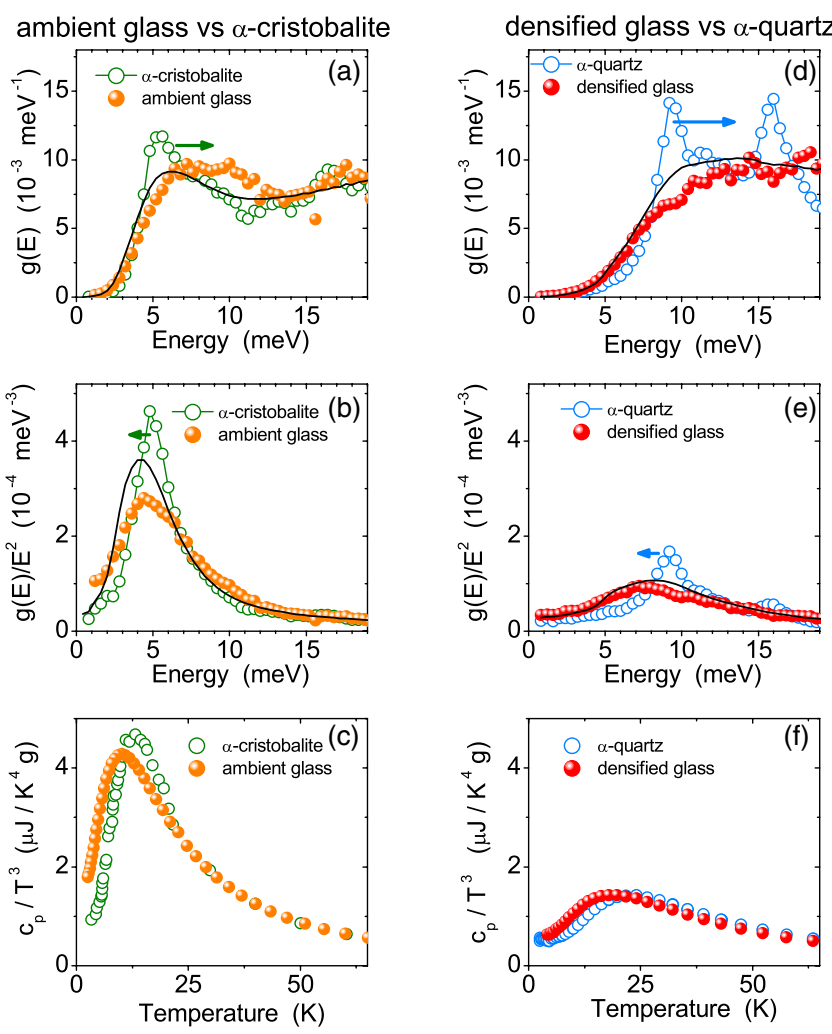

FIG. 4 (color online). Comparison of the density of states (a), (d) and the reduced density of states (b),(e) obtained with the NRAIXS, and of the specific heat (c),(f) for ambient silica glass and $\alpha$-cristobalite (a)-(c), and for densified silica glass and $\alpha$-quartz (d)-(f). The solid lines in (a)-(b), (d)-(e) show the modified DOS (a), (d) and reduced DOS (b),(e) of $\alpha$-cristobalite (a)(b) and $\alpha$-quartz (d)-(e) calculated allowing for the finite size of the Brillouin zone (see text). The arrows in (a)-(b), (d)-(e) emphasize the shift of the peaks in the DOS and reduced DOS of glasses relative to those of crystals. The experimental data for the specific heat of $\alpha$-cristobalite in (c) are those from Refs. [25,26].

silica glass [32] provides the same number of excess states [24]. Furthermore, an increase in the low-energy states of the studied crystals due to some kind of disorder is also excluded: The calculated DOS of ideal $\alpha$-cristobalite, $\alpha$ quartz, and coesite [24] contain nearly the same number of excess states.

Furthermore, Table 1 shows that the absolute number of all states $N_{\text {all }}$ in the considered energy regions is nearly the same for the glassy and crystalline pairs with matched density. Moreover, it is close to the fraction of the acoustic states of the corresponding crystal [33]. For $\alpha$-cristobalite the fraction of the acoustic states should be $8.333 \%$. Consistently, the number of states $N_{\text {all }}$ for both $\alpha$-cristobalite and ambient silica glass in the considered energy region is $8.4 \%$. For $\alpha$ quartz, the fraction of the acoustic states should be $11.111 \%$, and the number of states $N_{\text {all }}$ for $\alpha$-quartz and densified silica glass is $12.8 \%$ and $11.5 \%$, respectively.

These observations revise the role of disorder in the thermodynamics and atomic dynamics of glasses. They show that disorder does not influence much the energy spectrum of atomic vibrations. In the first approximation, the influence of disorder in the DOS is limited to the smearing out of the DOS features. Upon a closer view, disorder also separates in energy the peaks in the DOS and the reduced DOS. For crystals, they are located at the same energy. For glasses, the maximum of the DOS is shifted to higher energy, whereas the peak of the reduced DOS is shifted to lower energy [Figs. 4(a)-(b),(d)-(e)].

Before proceeding to discussion, we summarize the experimental observations and analyze what deserves an explanation. In terms of thermodynamics, the results show that the specific heat of the glasses and crystals with matched densities is essentially the same. This cancels the puzzle of the excessive specific heat of glasses for the studied system. In terms of atomic dynamics, the DOS of the glasses and corresponding crystals differ mainly by smoothening. In itself, this also looks natural and does not appeal for an explanation. However, the extensive studies of the boson peak define it as the puzzle and demand the explanation of the peak in the reduced DOS of the glasses.

In crystals, the peak in the reduced DOS is the acoustic van Hove singularity. It results from a piling up of the vibrational states near the boundary of the Brillouin zone. In our eyes, the striking qualitative and quantitative similarities in the DOS and reduced DOS for the glasses and corresponding crystals suggest that the boson peak is caused by the same mechanism. Indeed, similar to crystals, the dispersion relations in glasses reveal (see below for details) flattening near the boundary of the pseudo-Brillouin zone [34-36]. Furthermore, the studied glasses and corresponding crystals have nearly the same sound velocity and size of the Brillouin and pseudo-Brillouin zones [24]. Therefore, the dispersion curves for glasses and crystals should reveal the piling up effect at approximately the same energies. Consistently, the experimental results show that the boson peak and the van Hove singularity are located at 4.4 and $4.8 \mathrm{meV}$, respectively [low-density polymorphs, Fig. 4(b)], or at 7.4 and $9.2 \mathrm{meV}$, respectively [high-density polymorphs, Fig. 4(e)].

Near the boundary of the pseudo-Brillouin zone, the vibrational states in glasses (and liquids) are no longer the plane waves. The vibrational energy for a given wave vector is not a fixed value but reveals an energy spread [36-38]. This causes the difference in the dispersion curves related to the momentum-differential contributions to the DOS (current correlation function) and reduced DOS (dynamic structure factor). The dispersion curve related to the DOS is dominated by the upper edge of the energy spread. Therefore, while the dispersion curve related to the reduced DOS reveals the flattening near the border of the pseudo-Brillouin zone, the curve related to the DOS may continue to rise [39]. Accordingly, the DOS of the glasses does not show any special feature at the position of the boson peak, and reaches maximum at higher energy. 
This explains the discussed separation of the peak in the DOS and reduced DOS [Figs. 4(a)-(b),(d)-(e)].

The smoother appearance of the DOS in glasses can be attributed to the variance of the local structures and described by the finite width of the pseudo-Brillouin zone boundary [37,38]. We modified the measured DOS of $\alpha$ cristobalite and $\alpha$-quartz, allowing for the finite width of the pseudo-Brillouin zone boundary determined by the width of the first sharp diffraction peak in the static structure factor of glasses [24]. The results [Figs. 4(a)-(b),(d)-(e)] show that the modified DOS and reduced DOS of the crystals become similar to those of the corresponding glasses. Moreover, the smearing out reproduces the discussed separation of the peaks in the DOS and reduced DOS.

The plausible dominance of the piling up effect does not exclude other contributions. The shape of the DOS and reduced DOS is certainly influenced by an additional broadening caused by the finite lifetime of vibrational states [12]. Furthermore, it can be affected by a possible proximity to mechanical instabilities related to saddle points in the energy landscape [2] and invoked in the description of systems close to jamming [3-5]. Usually, the boson peak in jamming systems is discussed in terms of mechanical instability. We suspect that it also relates to the pileup effect, because jamming systems reveal a welldefined pseudo-Brillouin zone and show an excess of states even far away from the unjamming transition [5]. In this view, the mechanical instability would be mainly responsible not for the existence of the boson peak but for its shift to lower energy when approaching the unjamming transition. Therefore, the effects of instability most possibly should appear in the low-energy region of the DOS [40].

Finally, we note that the obtained results disentangle the origin of the anomalous thermal conductivity and specific heat of glasses: while the former is clearly caused by disorder, the latter is related to the lower density of the glasses. Thus, the low-energy (meV) dynamics and the lowtemperature $(\sim 10 \mathrm{~K})$ specific heat of the covalently-bonded glasses studied here are not anomalous: They are similar to those of crystals, and as such are more reflecting the residual order present in glasses than their structural disorder.

N. D. thanks the DFG (German Research Foundation) for financial support through the Heisenberg Program and the DFG Project DU 954-8/1. R. P. H. and D. B. thank the DFG for support through SPP1386. K. R. thanks EPSRC for use of the UK national supercomputing facility HECToR under Grant No. EP/F036809/1.

*Also at National Research Center "Kurchatov Institute," 123182 Moscow, Russia.chumakov@esrf.fr

${ }^{\dagger}$ Present address: European Synchrotron Radiation Facility, 6 rue Jules Horowitz, BP 220, F-38043 Grenoble Cedex 9, France.
${ }^{*}$ Also at Department of Earth Sciences, University College London, Gower St., London WC1E 6BT, UK.

${ }^{\S}$ Also at State Key Laboratory of Silicate Materials for Architecture, Wuhan University of Technology, Wuhan 430070, China.

[1] P. Flubacher, A. J. Leadbetter, J. A. Morrison, and B. P. Stoicheff, J. Phys. Chem. Solids 12, 53 (1959).

[2] T. Grigera, V. Martin-Mayor, G. Parisi, and P. Verrocchio, Nature (London) 422, 289 (2003).

[3] L. E. Silbert, A. J. Liu, and S. R. Nagel, Phys. Rev. Lett. 95, 098301 (2005).

[4] N. Xu, M. Wyart, A. J. Liu, and S. R. Nagel, Phys. Rev. Lett. 98, 175502 (2007).

[5] L. E. Silbert and M. Silbert, Phys. Rev. E 80, 041304 (2009).

[6] E. Duval, A. Boukenter, and T. Achibat, J. Phys. Condens. Matter 2, 10227 (1990).

[7] U. Tanaka, J. Phys. Soc. Jpn. 70, 1178 (2001).

[8] U. Buchenau, N. Nücker, and A. J. Dianoux, Phys. Rev. Lett. 53, 2316 (1984).

[9] C. A. Angell, Science 267, 1924 (1995).

[10] M. I. Klinger and A. M. Kosevich, Phys. Lett. A 280, 365 (2001).

[11] D. A. Parshin, H. R. Schober, and V. L. Gurevich, Phys. Rev. B 76, 064206 (2007).

[12] H. Shintani and Y. Tanaka, Nat. Mater. 7, 870 (2008).

[13] W. Schirmacher, G. Ruocco, and T. Scopigno, Phys. Rev. Lett. 98, 025501 (2007).

[14] F. Léonforte, A. Tanguy, J. P. Wittmer, and J.-L. Barrat, Phys. Rev. Lett. 97, 055501 (2006).

[15] G. Monaco and S. Mossa, Proc. Natl. Acad. Sci. U.S.A. 106, 16907 (2009).

[16] C. A. Angell, Y. Z. Yue, L.-M. Wang, J. R. D. Copley, S. Borick, and S. Mossa, J. Phys. Condens. Matter 15, S1051 (2003).

[17] W. Schirmacher, G. Diezemann, and C. Ganter, Phys. Rev. Lett. 81, 136 (1998).

[18] S. N. Taraskin, Y. L. Loh, G. Natarajan, and S. R. Elliott, Phys. Rev. Lett. 86, 1255 (2001).

[19] O. Pilla et al., J. Phys. Condens. Matter 16, 8519 (2004).

[20] A. I. Chumakov et al., Phys. Rev. Lett. 106, 225501 (2011).

[21] A. I. Chumakov, A. Q. R. Baron, R. Rüffer, H. Grünsteudel, H. F. Grünsteudel, and A. Meyer, Phys. Rev. Lett. 76, 4258 (1996).

[22] A. Bosak and M. Krisch, Phys. Rev. B 72, 224305 (2005).

[23] K. Refson, P. R. Tulip, and S. J. Clark, Phys. Rev. B 73, 155114 (2006).

[24] See Supplemental Material at http://link.aps.org/ supplemental/10.1103/PhysRevLett.112.025502 for the description of preparation and characterization of the samples, for the discussion of the experimental methods, data processing, theoretical calculations, comparison to neutron scattering data, and for the presentation of the raw and intermediate experimental and theoretical results.

[25] N. Bilir and W. A. Phillips, Philos. Mag. 32, 113 (1975).

[26] P. Richet, D. de Ligny and E. F. Westrum Jr., J. Non-Cryst. Solids 315, 20 (2003).

[27] G. N. Greaves, F. Meneau, O. Majerus, D. G. Jones, and J. Taylor, Science 308, 1299 (2005).

[28] G. N. Greaves, A. L. Greer, R. S. Lakes, and T. Rouxel, Nat. Mater. 10, 823 (2011).

[29] F. Birch, J. Geophys. Res. 66, 2199 (1961). 
[30] A. Monaco, A. I. Chumakov, Y.-Z. Yue, G. Monaco, L. Comez, D. Fioretto, W. A. Crichton, and R. Rüffer, Phys. Rev. Lett. 96, 205502 (2006).

[31] A. Monaco, A. I. Chumakov, G. Monaco, W. A. Crichton, A. Meyer, L. Comez, D. Fioretto, J. Korecki, and R. Rüffer, Phys. Rev. Lett. 97, 135501 (2006).

[32] E. Fabiani, A. Fontana, and U. Buchenau, J. Chem. Phys. 128, 244507 (2008).

[33] The calculated phonon dispersion relations [24] indeed show that for $\alpha$-cristobalite and $\alpha$-quartz, the considered regions contain mainly the acoustic modes, whereas for coesite they include also optical branches.

[34] B. Ruzicka, T. Scopigno, S. Caponi, A. Fontana, O. Pilla, P. Giura, G. Monaco, E. Pontecorvo, G. Ruocco, and F. Sette, Phys. Rev. B 69, 100201 (2004).
[35] E. L. Bove, E. Fabiani, A. Fontana, F. Paoletti, C. Petrillo, O. Pilla, and I. C. V. Bento, Europhys. Lett. 71, 563 (2005).

[36] H. R. Schober, J. Phys. Condens. Matter 16, S2659 (2004).

[37] V. M. Giordano and G. Monaco, Proc. Natl. Acad. Sci. U.S.A. 107, 21985 (2010).

[38] V. M. Giordano and G. Monaco, Phys. Rev. B 84, 052201 (2011).

[39] See Fig. 5(e) and the related discussion in Ref. [20].

[40] Note that the reduced DOS of ambient silica does not reach the Debye level down to $1.5 \mathrm{meV}$ [Fig. 1(c)], and this deviation is not described by the smearing out of the pseudo-Brillouin zone [Fig. 4(b)] suggesting other effects, e.g., mechanical instability or anharmonicity. 